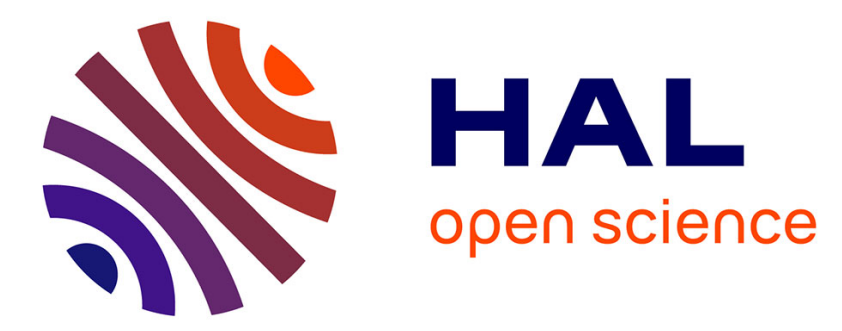

\title{
A unifying event-triggered control framework based on a hybrid small-gain theorem
}

Wei Wang, Dragan Nesic, Romain Postoyan, Maurice Heemels

\section{To cite this version:}

Wei Wang, Dragan Nesic, Romain Postoyan, Maurice Heemels. A unifying event-triggered control framework based on a hybrid small-gain theorem. 59th IEEE Conference on Decision and Control, CDC 2020, Dec 2020, Jeju Island, South Korea. hal-02934369

\section{HAL Id: hal-02934369 \\ https://hal.science/hal-02934369}

Submitted on 15 Sep 2020

HAL is a multi-disciplinary open access archive for the deposit and dissemination of scientific research documents, whether they are published or not. The documents may come from teaching and research institutions in France or abroad, or from public or private research centers.
L'archive ouverte pluridisciplinaire HAL, est destinée au dépôt et à la diffusion de documents scientifiques de niveau recherche, publiés ou non, émanant des établissements d'enseignement et de recherche français ou étrangers, des laboratoires publics ou privés. 


\title{
A unifying event-triggered control framework based on a hybrid small-gain theorem
}

\author{
W. Wang, D. Nešić, R. Postoyan, W.P.M.H. Heemels
}

\begin{abstract}
We propose a unifying emulation-based design framework for the event-triggered control of nonlinear systems that is based on a hybrid small-gain perspective. We show that various existing event-triggered controllers fit the unifying perspective. Moreover, we demonstrate that the flexibility offered by our approach can be used for the development of novel event-triggered schemes and for a systematic modification and improvement of existing schemes. Finally, we illustrate via a simulation example that these novel and/or modified eventtriggered controllers can lead to a reduction in the required number of transmissions, while still guaranteeing the same stability properties.
\end{abstract}

\section{INTRODUCTION}

Event-triggered control (ETC) is a class of sampled-data schemes where the sampler and hold devices are triggered whenever a state or output-dependent condition holds [3], [4], [14]. As such, ETC is a natural generalization of the classical sampled-data control [7], since state-dependent sampling is used instead of equidistant sampling. Reasons for considering ETC are manifold and we single out the motivation arising from the emerging resource-aware control applications with packed-based communication networks, where the design of the event-triggering condition can be used to reduce the communication between the plant and controller while maintaining appropriate performance and robustness guarantees, see, e.g., [8], [12], [20], [21], [24], [26], [28]. This approach was experimentally verified for mobile robots [22] and for vehicle platooning [9], for instance.

The benefits of ETC were recognised for a long time, see [4], and many approaches have been proposed in the literature since then. However, the relationship between the various approaches, the intuition behind different schemes and their advantages/disadvantages are hard to understand as the underlying design tools and philosophy appear to be different. Typically, it is often unclear how the design flexibility within each approach affects the required transmissions and system performance. The recent studies on the required transmissions [16], [23] for classes of ETC systems shed some light on this issue, but a systematic design framework remains elusive.

This work is supported by the Australian Research Council under the Discovery Project DP170104099, HANDY project ANR-18CE40-0010-02 and the France-Australia collaboration project IRPARS CNRS. W. Wang and D. Nešić are with the Department of Electrical and Electronic Engineering, The University of Melbourne, Parkville, Australia, wei.wang@unimelb.edu.au; dnesiceunimelb. edu. au.

R. Postoyan is with the Universite de Lorraine, CNRS, CRAN, F-54000 Nancy, France, romain.postoyan@univ-lorraine.fr

W.P.M.H. Heemels is with the Dept. Mechanical Engineering, Eindhoven University of Technology, The Netherlands, m. heemels@tue.nl
The purpose of this paper is to show that a large class of seemingly unrelated emulation-based ETC schemes can be unified within a novel design framework. This provides valuable insights not previously observed in the literature. One advantage of such a unifying design perspective is that it provides clear viewpoints on the essential differences and similarities of existing ETC strategies. It also provides the design flexibility for each existing approach, which allows for a systematic modification or "redesign" of existing schemes. Moreover, our approach can be used as a design framework for generating novel ETC schemes, not previously considered in the literature. This design flexibility enables a more systematic design methodology that may be used for the reduction of required transmissions as we illustrate via a numerical example.

This work focuses on the emulation based approach, see the relevant results in e.g., [8], [12], [20], [21], [24], [26], [28] and the references therein, which is a prevailing design approach in ETC. Emulation design consists of two steps. In the first step, a (potentially dynamic) continuous-time controller is designed to robustly stabilize the continuoustime plant. In the second step, the controller is implemented via an event-triggering rule that might involve auxiliary state variables, see, e.g., [8], [12], [24], while preserving important stability and performance properties. Our approach is to decompose the closed-loop system as an interconnection of two hybrid systems that need to satisfy a recently proposed hybrid small-gain theorem [17] to ensure stability. The required small-gain condition can be ensured by a judicious selection of auxiliary variables, as well as the design of the event-triggering rule. We demonstrate that this smallgain analysis underlies various important emulation-based schemes of the literature [12], [24], [26] and provides a flexible design framework for the analysis of existing and the design of novel ETC schemes. Indeed, the analysis reveals a singularity: the ISS gain of one of the subsystems is equal to zero, which trivially ensures the satisfaction of the small-gain condition. Based on this observation, we redesign the original techniques in [12], [24], [26] so that this is no longer the case, thereby enlarging the flow set, which may help reducing the number of transmissions as illustrated on a numerical example.

Note that a small-gain interpretation of the static triggering rule in [26] was already demonstrated in [17, Section V.C]. Here, we generalize this approach here to a much larger class of dynamic triggering rules that involve auxiliary state variables. The proofs are omitted for space reasons. 


\section{PREliminaries}

We will model closed-loop ETC systems as hybrid systems [13] of the form below, for which the jump times correspond to triggering instants,

$$
\mathcal{H}\left\{\begin{aligned}
\dot{q} & =\mathcal{F}(q) & & q \in C \\
q^{+} & =\mathcal{G}(q) & & q \in D
\end{aligned}\right.
$$

where $q \in \mathbb{R}^{n}$ is the state, $C, D \subseteq \mathbb{R}^{n}$ are the flow and the jump sets, which are assumed to be closed, and $\mathcal{F}, \mathcal{G}$ : $\mathbb{R}^{n} \rightarrow \mathbb{R}^{n}$ are continuous. Hence, system (1) satisfies the hybrid basic conditions in [13]. We focus on the following stability property, and we omit the other description of the notation and definitions used throughout this paper and refer the reader to Section II in [27].

Definition 1: System $\mathcal{H}$ is uniformly globally asymptotically stable $(U G A S)$, if there exists $\beta \in \mathcal{K} \mathcal{L}$ such that, for any solution $\varphi$ and $(t, j) \in \operatorname{dom} \varphi$, it holds that

$$
|\varphi(t, j)| \leq \beta(|\varphi(0,0)|, t+j),
$$

and all maximal solutions are complete.

Definition 2: System $\mathcal{H}$ has a uniform semi-global dwell time outside the origin, if for any $\Delta>0$, there exists $\tau(\Delta) \in$ $\mathbb{R}_{>0}$ such that, for any hybrid solution $\varphi$ with $|\varphi(0,0)| \leq \Delta$ and for all $j \in \mathbb{Z}_{>0}$,

$$
\left(\forall t \in\left[t_{j}, t_{j+1}\right], \quad \varphi(t, j) \neq 0\right) \Rightarrow\left(t_{j+1}-t_{j} \geq \tau(\Delta)\right),
$$

where $\operatorname{dom} \varphi=\bigcup\left[t_{j}, t_{j+1}\right] \times\{j\}$.

Remark 1: The only hybrid state, on which the existence of a dwell-time is not guaranteed by the event-triggering conditions in this paper, is the origin. The reason that we do not "worry" about the origin is the fact that solutions initialized at the origin are not unique for the considered systems in the sequel: they all remain at the origin for all future hybrid times but their hybrid time domains can take any form, ranging from Zeno behaviour to no jump at all, see also [24]. Hence, there exist (infinitely many) zero solutions, whilst might not have a dwell-time.

\section{HYBRID MODEL}

The purpose of this section is to derive a hybrid model of a large class of emulation-based ETC schemes, like in [24], and to recall a small-gain theorem for hybrid systems from [17] that can enable a unifying ETC design framework. We consider the plant

$$
\dot{x}_{p}=f_{p}\left(x_{p}, u\right),
$$

where $x_{p} \in \mathbb{R}^{n_{p}}$ is the state. The first step in the emulation approach is to design a controller

$$
\dot{x}_{c}=f_{c}\left(x_{c}, x_{p}\right) \quad u=g_{c}\left(x_{c}, x_{p}\right),
$$

to robustly stabilize (4) as formalised below, see Assumption 1. We have that $u \in \mathbb{R}^{n_{u}}$ and $x_{c} \in \mathbb{R}^{n_{c}}$ are respectively the control input and the state of the controller. For a static feedback controller, (5) simplifies to $u=g_{c}\left(x_{p}\right)$. The functions $f_{p}$ and $f_{c}$ are assumed to be continuous, and $g_{c}$ is assumed to be continuously differentiable and zero at zero.

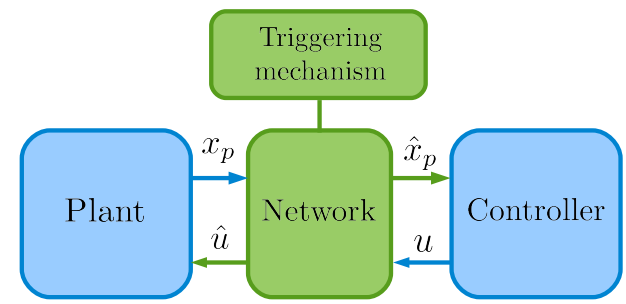

Fig. 1: Block diagram of the setup

In the second step of emulation, we implement the designed controller (5) over a network, see Fig. 1. The communication occurs at transmission instants $t_{j}, j \in \mathcal{I} \subseteq \mathbb{Z}_{\geq 0}$ at which the current values of $x_{p}$ and $u$ are sent over the network. In this setup, plant (4) has no longer access to $u$, but to its networked version $\hat{u}$, and controller (5) has access to $\hat{x}_{p}$, the networked version of $x_{p}$. We assume ideal packedbased communication in the sense that $\hat{x}_{p}\left(t_{j}^{+}\right)=x_{p}\left(t_{j}\right)$ and $\hat{u}\left(t_{j}^{+}\right)=u\left(t_{j}\right)$, for any $j \in \mathcal{I}$. Between two successive transmission instants, $\hat{x}_{p}$ and $\hat{u}$ are governed by the dynamics of the holding devices

$$
\left.\begin{array}{rl}
\dot{\hat{x}}_{p} & =\hat{f}_{p}\left(x_{p}, \hat{x}_{p}, \hat{u}\right) \\
\dot{\hat{u}} & =\hat{f}_{c}\left(x_{c}, \hat{x}_{p}, \hat{u}\right)
\end{array}\right\} \quad t \in\left(t_{j}, t_{j+1}\right), j \in \mathcal{I},
$$

where $\hat{f}_{p}$ and $\hat{f}_{c}$ are continuous. Zero-order-hold devices correspond to $\hat{f}_{p}=\hat{f}_{c}=0$, for instance.

Let $x:=\left(x_{p}, x_{c}\right) \in \mathbb{R}^{n_{x}}, n_{x}:=n_{p}+n_{c}$, denote the concatenation of the plant and the controller state, and let $e:=\left(e_{x_{p}}, e_{u}\right) \in \mathbb{R}^{n_{e}}, n_{e}:=n_{p}+n_{u}$, denote the networkinduced error with $e_{x_{p}}:=\hat{x}_{p}-x_{p}$ and $e_{u}:=\hat{u}-u$. We then derive that $\dot{x}=f(x, e)$ with $f$ being determined by (4)-(6), where $e$ is an input to $x$-system. We assume that the controller (5) is designed so that the following conditions are satisfied.

Assumption 1: There exist a continuously differentiable function $V: \mathbb{R}^{n_{x}} \rightarrow \mathbb{R}_{\geq 0}$, and functions $\underline{\alpha}_{V}, \bar{\alpha}_{V}, \alpha_{V}, \gamma \in$ $\mathcal{K}_{\infty}$ such that the following hold.

(i) For all $x \in \mathbb{R}^{n_{x}}, \underline{\alpha}_{V}(|x|) \leq V(x) \leq \bar{\alpha}_{V}(|x|)$.

(ii) For all $x \in \mathbb{R}^{n_{x}}$ and $e \in \mathbb{R}^{n_{e}},\langle\nabla V(x), f(x, e)\rangle \leq$ $-\alpha_{V}(|x|)+\gamma(|e|)$.

In general, the transmission instants $t_{j}, j \in \mathcal{I}$, are generated by an event-triggering mechanism, which may depend on $x_{p}, u$ and some auxiliary variables $\eta \in \mathbb{R}^{n_{\eta}}$, $n_{\eta} \in \mathbb{Z}_{>0}$ that are modeled as

$$
\begin{aligned}
& \dot{\eta}=h(x, e, \eta), \quad t \in\left(t_{j}, t_{j+1}\right), j \in \mathcal{I}, \\
& \eta\left(t_{j}^{+}\right)=\ell\left(x\left(t_{j}\right), e\left(t_{j}\right), \eta\left(t_{j}\right)\right),
\end{aligned}
$$

where $h, \ell: \mathbb{R}^{n_{x}} \times \mathbb{R}^{n_{e}} \times \mathbb{R}^{n_{\eta}} \rightarrow \mathbb{R}^{n_{\eta}}$ are continuous functions to be designed. The overall closed-loop ETC system is then modeled as the hybrid system

$$
\left.\begin{array}{l}
\dot{x}=f(x, e) \\
\dot{e}=g(x, e) \\
\dot{\eta}=h(x, e, \eta)
\end{array}\right\} \quad(x, e, \eta) \in C
$$




$$
\left.\begin{array}{rl}
x^{+} & =x \\
e^{+} & =0 \\
\eta^{+} & =\ell(x, e, \eta)
\end{array}\right\} \quad(x, e, \eta) \in D
$$

where functions $f$ and $g$ can be derived from (4)-(6), whereas the sets $C, D \subset \mathbb{R}^{n_{x}} \times \mathbb{R}^{n_{e}} \times \mathbb{R}^{n_{\eta}}$ and functions $h, \ell$ are to be designed and they lead to different event-triggering conditions. To design $C, D, h$ and $\ell$, we interpret system (8) as a feedback interconnection of the $(x, \eta)$-system and the $e$-system, see Fig. 2, and rewrite the model as

$$
\begin{aligned}
& \mathcal{H}_{1}\left\{\begin{array}{rr}
(\dot{x}, \dot{\eta})=\mathcal{F}_{1}(q) & q \in C \\
\left(x^{+}, \eta^{+}\right)=\mathcal{G}_{1}(q) & q \in D
\end{array}\right. \\
& \mathcal{H}_{2}\left\{\begin{array}{cc}
\dot{e}=\mathcal{F}_{2}(q) & q \in C \\
e^{+}=\mathcal{G}_{2}(q) & q \in D,
\end{array}\right.
\end{aligned}
$$

where $q:=(x, e, \eta), \mathcal{F}_{1}(q):=(f(x, e), h(x, e, \eta))$, $\mathcal{G}_{1}(q):=(x, \ell(x, e, \eta)), \mathcal{F}_{2}(q):=g(x, e), \mathcal{G}_{2}(q):=0$.

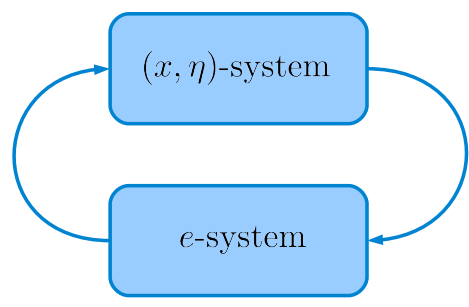

Fig. 2: Block diagram of the decomposition

The feedback interconnection (10) provides a starting point for our unifying emulation-based ETC design framework. The design consists in shaping the sets $C$ and $D$ of hybrid system (10) (equivalently of the system (8)) together with defining the flow and jumps dynamics of $\eta$, if any, to guarantee that a hybrid small-gain condition holds. We next state the hybrid small-gain theorem from [17, Theorem III.3], which is a crucial technical tool in our approach.

Proposition 1: Suppose that there exist locally Lipschitz functions $V_{i}: \mathbb{R}^{n_{i}} \rightarrow \mathbb{R}_{\geq 0}, n_{1}:=n_{x}+n_{\eta}, n_{2}:=n_{e}$, $\underline{\alpha}_{i}, \bar{\alpha}_{i} \in \mathcal{K}_{\infty}, \chi_{i} \in \mathcal{K}_{\infty} \cup\{0\}$, and positive definite functions $\alpha_{i}: \mathbb{R}_{\geq 0} \rightarrow \mathbb{R}_{\geq 0}, i \in\{1,2\}$, such that the following hold.

(i) For all $q \in C \cup D \underline{\alpha}_{1}(|(x, \eta)|) \leq V_{1}(x, \eta) \leq$ $\bar{\alpha}_{1}(|(x, \eta)|)$, and $\underline{\alpha}_{2}(|e|) \leq V_{2}(e) \leq \bar{\alpha}_{2}(|e|)$.

(ii) For all $q \in C$,

$$
\begin{aligned}
V_{1}(x, \eta) & \geq \chi_{1}\left(V_{2}(e)\right) \\
& \Rightarrow V_{1}^{\circ}\left((x, \eta) ; \mathcal{F}_{1}(q)\right) \leq-\alpha_{1}(|(x, \eta)|), \\
V_{2}(e) & \geq \chi_{2}\left(V_{1}(x, \eta)\right) \\
& \Rightarrow V_{2}^{\circ}\left(e ; \mathcal{F}_{2}(q)\right) \leq-\alpha_{2}(|e|) .
\end{aligned}
$$

(iii) For all $q \in D, V_{i}\left(\mathcal{G}_{i}(q)\right) \leq V_{i}(q), i \in\{1,2\}$.

(iv) The small-gain condition $\chi_{1} \circ \chi_{2}(s)<s$ holds for all $s>0$.

Then, there exist $\bar{\alpha}_{U}, \underline{\alpha}_{U}, \rho \in \mathcal{K}_{\infty}$ and positive definite functions $\alpha_{U}: \mathbb{R}_{\geq 0} \rightarrow \mathbb{R}_{\geq 0}$ such that the following holds for $U(q):=\max \left\{V_{1}(q), \rho\left(V_{2}(q)\right)\right\}$.

(a) For all $q \in C \cup D, \underline{\alpha}_{U}(|q|) \leq U(q) \leq \bar{\alpha}_{U}(|q|)$. (b) For all $q \in C, U^{\circ}(q ; \mathcal{F}(q)) \leq-\alpha_{U}(|q|)$, where $\mathcal{F}(q):=$ $\left(\mathcal{F}_{1}(q), \mathcal{F}_{2}(q)\right)$.

(c) For all $q \in D, U(\mathcal{G}(q)) \leq U(q)$, where $\mathcal{G}(q):=$ $\left(\mathcal{G}_{1}(q), \mathcal{G}_{2}(q)\right)$.

Proposition 1 can be used to construct a Lyapunov function for system (10) if its $(x, \eta)$-system and $e$-systems satisfy input-to-state (ISS) properties, see its definition in hybrid system setting in [6], with respect to inputs $e$ and $(x, \eta)$, as specified in the ISS Lyapunov based conditions (i)-(iii) in combination with the small-gain conditions (iv). We will show how these ISS conditions can be ensured through the ETC designs of [12], [24], [26] in the following sections. Uniform global asymptotic stability of system (10) then follows from (a)-(c) of Proposition 1 under extra conditions on the hybrid time domains of its solutions, see [24, Theorem 1], as stated next.

Theorem 1: Consider system (10) and suppose that the following holds.

1) The conditions of Proposition 1 are verified.

2) The system has a uniform semi-global dwell time outside the origin.

3) Maximal solutions are complete.

Then, the system is UGAS.

Novelties of our approach are: (i) we demonstrate that various popular ETC schemes in the literature can be reinterpreted using this unifying framework based on a hybrid small-gain view; (ii) we show that the original triggering conditions can be systematically modified to potentially generate longer inter-event times. We sometimes use the same notation to denote different quantities from one section to another in the sequel to underline the similarities among the different triggering techniques.

\section{THE RELATIVE THRESHOLD TECHNIQUE [26]}

We start with the technique proposed in [26], which was exploited and extended in various other contexts in, e.g., [1], [2], [8], [10], [11], [17], [18].

\section{A. Model}

This approach does not involve auxiliary variable $\eta$, hence (8) reduces to

$$
\left.\begin{array}{c}
\begin{array}{c}
\dot{x}=f(x, e) \\
\dot{e}=g(x, e)
\end{array} \\
x^{+}=x \\
e^{+}=0
\end{array}\right\} \quad q \in C,
$$

where $q:=(x, e)$. We translate the considered triggering condition in [26] as sets $C$ and $D$ given by

$$
\begin{aligned}
& C=\left\{(x, e): \gamma(|e|) \leq \sigma \alpha_{V}(|x|)\right\} \\
& D=\left\{(x, e): \gamma(|e|) \geq \sigma \alpha_{V}(|x|)\right\},
\end{aligned}
$$

see also, e.g. [5], [24], where $\sigma \in(0,1)$ is a design parameter, and $\alpha_{V}, \gamma \in \mathcal{K}_{\infty}$ come from Assumption 1. We need the next Lipschitz conditions in view of [26, Theorem III.1], to guarantee the existence of a uniform semi-global dwell time outside the origin, see also [24, Theorem 4].

Assumption 2: The functions $f, g$ in (11), and $\alpha_{V}^{-1}, \gamma \in$ $\mathcal{K}_{\infty}$ from Assumption 1 are locally Lipschitz. 


\section{B. Analysis}

In this case, we can show that the conditions of Proposition 1 hold with gains $\chi_{1}$ and $\chi_{2}$ given by

$$
\begin{aligned}
& \chi_{1}(s)=0 \\
& \chi_{2}(s)=(1+\varepsilon)\left(\gamma^{-1} \circ\left(\sigma \alpha_{V} \circ \underline{\alpha}_{V}^{-1}(s)\right)\right)^{2},
\end{aligned}
$$

where $\varepsilon>0$ can take any value, and $\gamma, \alpha V, \underline{\alpha}_{V}$ come from Assumption 1.

According to Theorem 1, we need two extra conditions to conclude about the stability of the origin: the dwelltime property and the completeness of maximal solutions. Thanks to Assumption 2, as shown in [26, Theorem III.1] and [24, Theorem 4], system (11)-(12) has a uniform semiglobal dwell time outside the origin. Moreover, according to [24, Theorem 4], all maximal solutions to system (11)-(12) are complete. Then, we can apply Theorem 1 to derive the following stability result.

Corollary 1: Consider system (11)-(12) and suppose that Assumptions 1 and 2 hold. Then, the system is UGAS.

This implies that the relative threshold technique [26] fits our framework. It is important to note that the gain $\chi_{1}$ in (13) is zero. This suggests that we can modify the triggering condition, i.e., the flow and the jump sets, in such a way that the ISS property of the $x$-system holds with a nonzero gain, while still preserving the small-gain condition and thus stability. By doing so, we inflate the flow set $C$, which can help generating longer inter-event times as shown on an example in Section VII. This is explained in the sequel.

Remark 2: Note that [17] already related the relative threshold technique [26] with a hybrid Lyapunov small-gain theorem. The difference here is that we consider exactly the same condition as in the original paper [26], and not a modified, more conservative, version as in [17].

\section{Redesign}

We modify the triggering condition by defining

$$
\begin{aligned}
& C=\left\{(x, e):(1-\delta) \gamma(|e|) \leq \sigma \alpha_{V}(|x|)\right\} \\
& D=\left\{(x, e):(1-\delta) \gamma(|e|) \geq \sigma \alpha_{V}(|x|)\right\},
\end{aligned}
$$

where $\delta \gamma(|e|)$ is the newly introduced term enlarging the flow set compared to (12). To design $\delta$, we rely on the next assumption, which is satisfied when Assumption 1 holds with $\underline{\alpha}_{V}, \bar{\alpha}_{V}, \alpha_{V}$ all quadratic or all linear for instance.

Assumption 3: There exists $c>0$ such that for any $s \geq 0$, $\alpha_{V} \circ \underline{\alpha}_{V}^{-1}(s) \leq c \alpha_{V} \circ \bar{\alpha}_{V}^{-1}(s)$.

We select the parameter $\delta$ in $\left(0, \frac{1-\sigma}{1-\sigma+\sigma c}\right)$ by verifying conditions of Proposition 1. As a result, Proposition 1 holds with

$$
\begin{aligned}
& \chi_{1}(s)=\bar{\alpha}_{V} \circ \alpha_{V}^{-1}\left(\frac{\delta}{\nu(1-\sigma)} \gamma\left(s^{\frac{1}{2}}\right)\right) \\
& \chi_{2}(s)=\left(\gamma^{-1}\left((1+\varepsilon) \bar{\chi}_{2}(s)\right)\right)^{2},
\end{aligned}
$$

where $\nu \in(0,1)$ and $\varepsilon>0$ are some constants, and $\bar{\chi}_{2}(s)=$ $\frac{\sigma}{1-\delta} \alpha_{V} \circ \underline{\alpha}_{V}^{-1}(s)$

Then, we derive the UGAS property of system (11), (14) by following the analysis in Section IV-B.
Corollary 2: Consider system (11), (14) and suppose that Assumptions 1, 2 and 3 hold. Then, the system is UGAS.

\section{THE DYNAMIC TRIGGERING CONDITION OF [12]}

Here we study the dynamic event-triggering rule of [12], which has been extended afterwards to other (distributed) event-triggered control setting, see, e.g., [8].

\section{A. Model}

The auxiliary variable has the next dynamics [12]

$$
\begin{aligned}
\dot{\eta} & =-\beta(\eta)+\sigma \alpha_{V}(|x|)-\gamma(|e|) & & q \in C \\
\eta^{+} & =\eta & & q \in D
\end{aligned}
$$

where $\beta: \mathbb{R}_{\geq 0} \rightarrow \mathbb{R}_{\geq 0}$ is any continuous positive definite function. The triggering technique of [12] leads to

$$
\begin{aligned}
& C=\left\{q: \eta+\theta\left(\sigma \alpha_{V}(|x|)-\gamma(|e|)\right) \geq 0, \eta \geq 0\right\} \\
& D=\left\{q: \eta+\theta\left(\sigma \alpha_{V}(|x|)-\gamma(|e|)\right) \leq 0, \eta \geq 0\right\},
\end{aligned}
$$

for $\alpha_{V}, \gamma \in \mathcal{K}_{\infty}$ from Assumption $1, \sigma \in(0,1)$ and $\theta>0$.

\section{B. Analysis}

Here, the conditions of Proposition 1 hold with

$$
\chi_{1}(s)=0, \quad \chi_{2}(s)=\left(\gamma^{-1}\left((1+\varepsilon) \bar{\chi}_{2}(s)\right)\right)^{2},
$$

where $\bar{\chi}_{2}(s)=\left(\frac{s}{\theta}+\sigma \alpha_{V} \circ \underline{\alpha}_{V}^{-1}(s)\right)$ for any $\varepsilon>0$.

Moreover, the system (8), (16), (17) admits a uniform semi-global dwell time outside the origin when Assumption 2 holds, according to [12, Proposition 2.3]. This, with completeness of maximal solutions, ensures the following property.

Corollary 3: Consider system (8), (16), (17) and suppose that Assumptions 1 and 2 hold. The system is UGAS.

Note that $\chi_{1}$ is equal to zero, as in Section IV. We can modify the flow dynamics of $\eta$, as well as the flow and the jump sets, so that $\chi_{1}$ is no longer zero, while still ensuring the small-gain condition and thus stability.

\section{Redesign}

The redesign of [12] presented here relies on the next condition, in place of item (ii) of Assumption 1, which can always be satisfied by modifying $V$ in Assumption 1 (which is supposed to hold) if needed, in view of [15, Theorem 2]. We thus consider $a_{V}>0$ and $\gamma \in \mathcal{K}_{\infty}$ such that, for all $x \in \mathbb{R}^{n_{x}}$ and $e \in \mathbb{R}^{n_{e}}$,

$$
\langle\nabla V(x), f(x, e)\rangle \leq-a_{V} V(x)+\gamma(|e|) .
$$

We modify the dynamics of $\eta$ to

$$
\begin{aligned}
\dot{\eta} & =-b \eta+\sigma \alpha_{V} V(x)-(1-\delta) \gamma(|e|) & & q \in C, \\
\eta^{+} & =\eta & & q \in D,
\end{aligned}
$$

where $b>0$ and $\delta \in(0,1)$ are to be designed. We have taken $\beta$ in (16) linear in (20) for the sake of convenience. The main difference with (16) is that we have added the term $\delta \gamma(|e|)$ in the flow map of $\eta$, which slows down the decrease 
of $\eta$ and which may thus help reducing the number of jumps. We modify the flow and the jump sets accordingly as

$$
\begin{aligned}
& C=\left\{q: \eta+\theta\left(\sigma a_{V} V(x)-(1-\delta) \gamma(|e|)\right) \geq 0, \eta \geq 0\right\} \\
& D=\left\{q: \eta+\theta\left(\sigma a_{V} V(x)-(1-\delta) \gamma(|e|)\right) \leq 0, \eta \geq 0\right\} .
\end{aligned}
$$

We design $\delta$ to ensure that the conditions of Proposition 1 hold. In particular, we take

$$
\delta \in\left(0, \frac{a_{1}}{a_{1}+a_{2}}\right),
$$

where $a_{1}:=\min \left\{(1-\sigma) a_{V}, b\right\}$ and $a_{2}:=\max \left\{\theta^{-1}, \sigma a_{V}\right\}$. As a result, conditions of Proposition 1 hold with

$$
\chi_{1}(s)=\frac{\delta}{\nu a_{1}} \gamma\left(s^{\frac{1}{2}}\right), \quad \chi_{2}(s)=\left(\gamma^{-1}\left(\frac{1+\varepsilon}{1-\delta} a_{2} s\right)\right)^{2},
$$

for some $\nu \in(0,1)$ and $\varepsilon>0$.

We can then derive, similarly to Corollary 3 , the next stability result. Note that, for any given initial condition, the first jump time generated by system (8), (20), (21) occurs later than the one generated by (8), (16), (17). Using this argument, we can prove that system (8), (20), (21) has a semiglobal dwell-time outside the origin; completeness of maximal solutions follows like for Corollary 3.

Corollary 4: Consider system (8), (20), (21) and suppose that Assumptions 1 and 2 hold. The system is UGAS.

\section{Decreasing threshold STRATEgy [24]}

We next consider the decreasing threshold strategy of [24, Section V.A], see similar techniques in, e.g., [19], [25].

\section{A. Model}

In [24, Section V.A], a scalar variable $\eta$ is used in the triggering condition. Its dynamics are given by

$$
\begin{aligned}
\dot{\eta} & =-\beta(\eta) & q \in C, \\
\eta^{+} & =\eta & q \in D,
\end{aligned}
$$

where $\beta$ is any function in $\mathcal{K}_{\infty}$,

$$
\begin{aligned}
& C:=\left\{q: \gamma(|e|) \leq \sigma\left(\alpha_{V}(|x|)+\beta(\eta)\right), \quad \eta \geq 0\right\} \\
& D:=\left\{q: \gamma(|e|) \geq \sigma\left(\alpha_{V}(|x|)+\beta(\eta)\right), \quad \eta \geq 0\right\},
\end{aligned}
$$

$\sigma \in(0,1)$ is a design parameter and $\gamma, \alpha_{V}$ come from Assumption 1. Note that we have slightly modified the original triggering condition of [24, Section V.A] in (24), but the rationale remains the same.

\section{B. Analysis}

We verify the conditions of Proposition 1, which hold with

$$
\chi_{1}(s)=0, \quad \chi_{2}(s)=\left(\gamma^{-1}\left((1+\varepsilon) \sigma\left(\alpha_{V} \circ \underline{\alpha}_{V}^{-1}+\beta\right)\right)\right)^{2},
$$

where $\varepsilon>0$ is any strictly positive constant.

By following similar arguments as in [24, Section V.A], we have that system (8) with (23) and (24) has a uniform semi-global dwell time outside the origin, and its maximal solutions are complete. Consequently, we have the next result.

Corollary 5: Consider the system (8) with (23) and (24) and suppose that Assumptions 1 and 2 hold. The system is UGAS.

\section{Redesign}

We redesign the technique presented above. As in Section $\mathrm{V}-\mathrm{C}$, we rely on (19). We now consider

$$
\begin{aligned}
\dot{\eta} & =-b \eta+\delta \gamma(|e|) & q & \in C, \\
\eta^{+} & =\eta & q & \in D,
\end{aligned}
$$

where $b>0$ and $\delta>0$ are parameters to be designed. Note the major difference exists between (23) and (25). While in (23), $\eta$ is an open-loop signal and this is no longer the case in (25) as the flow dynamics now depends on $|e|$, via the term $\delta \gamma(|e|)$.

The flow and the jump sets are given by

$$
\begin{aligned}
& C:=\left\{q: \gamma(|e|) \leq \sigma\left(a_{V} V(x)+b \eta\right), \quad \eta \geq 0\right\} \\
& D:=\left\{q: \gamma(|e|) \geq \sigma\left(a_{V} V(x)+b \eta\right), \quad \eta \geq 0\right\},
\end{aligned}
$$

where $a_{V}$ and $b$ come from (19) and (25), respectively.

We select $\delta$ in $(25)$ such that

$$
\delta \in\left(0, \frac{1-\sigma}{\sigma} \frac{a_{1}}{a_{2}}\right),
$$

where $a_{1}:=\min \left\{a_{V}, b\right\}$ and $a_{2}:=\max \left\{a_{V}, b\right\}$. As a result, the conditions of Proposition 1 are satisfied with

$\chi_{1}(s)=\frac{\delta}{\nu(1-\sigma) a_{1}} \gamma\left(s^{\frac{1}{2}}\right), \chi_{2}(s)=\left(\gamma^{-1}\left((1+\varepsilon) \sigma a_{2} s\right)\right)^{2}$,

for some constants $\nu \in(0,1)$ and $\varepsilon>0$. By following similar arguments as in the previous sections, we derive the next stability result from Section VI-B.

Corollary 6: Consider system (8), (25), (26) and suppose that Assumptions 1 and 2 hold. The system is UGAS.

\section{NUMERICAL EXAMPLE}

We compare the redesigned techniques presented in Sections IV-C, V-C and VI-C, respectively, with the corresponding original triggering conditions, in terms of number of transmissions. We consider for this purpose the linear example in [26] given by $\dot{x}=A x+B u$, where $A=$ $\left[\begin{array}{cc}0 & 1 \\ -2 & 3\end{array}\right], B=\left[\begin{array}{cc}0 & -1\end{array}\right]^{\top}$. Controller (5) is taken as $u=K x$ with $K:=\left[\begin{array}{ll}-3 & -7\end{array}\right]$. Assumption 1 is verified with $V(x)=x^{\top} P x, P=\left[\begin{array}{cc}0.55 & -0.5 \\ -0.5 & 0.75\end{array}\right], \underline{\alpha}_{V}(s)=$ $\lambda_{\min }(P) s^{2}, \bar{\alpha}_{V}(s)=\lambda_{\max }(P) s^{2}, \alpha_{V}(s)=\frac{1}{2} s^{2}$ and $\gamma(s)=$ $2|P B K| s^{2}$ for any $s \geq 0$. On the other hand, Assumption 3 is satisfied with $c=\lambda_{\max }(P) / \lambda_{\min }(P)$ and the inequality in (19) holds with $a_{V}=1 /\left(2 \lambda_{\max }(P)\right)$.

We have selected the parameters as $\sigma=0.9, \delta=$ $0.9 \frac{1-\sigma}{1-\sigma+\sigma c}$ in (14), $\delta=0.9 \times 0.0413$ in (22), $\delta=0.999 \times$ 0.0479 in (27), and $\theta=1$. The dynamic triggering condition of [12] studied in Section V has been implemented with $\dot{\eta}=$ $-\eta+\sigma \alpha_{V} V(x)-\gamma(|e|)$ and $C$ and $D$ defined accordingly so that we can analyze the impact of the redesign condition presented in Section V-C; hence, $\beta(\eta)=b \eta=\eta$ for any $\eta \geq 0$. We have similarly simulated the triggering conditions of Section VI using (26) for both cases with $\sigma=0$ and $\sigma=0.9$, with $\delta$ in (27) and $\beta(\eta)=b \eta=\eta$ for any $\eta \geq 0$. 


\begin{tabular}{ccccccc}
\hline & \multicolumn{2}{c}{ Section IV } & \multicolumn{2}{c}{ Section V } & \multicolumn{2}{c}{ Section VI } \\
& {$[26]$} & Redesign & {$[12]$} & Redesign & {$[24]$} & Redesign \\
\hline$\tau_{\text {avg }}$ & 0.0738 & 0.0748 & 0.2723 & 0.2830 & 0.2330 & 0.2410 \\
$\tau_{\text {min }}$ & 0.0246 & 0.0248 & 0.0176 & 0.0180 & 0.0150 & 0.0150 \\
\hline
\end{tabular}

TABLE I: Average $\left(\tau_{\text {avg }}\right)$ and minimum $\left(\tau_{\min }\right)$ inter-transmission times over 10 different initial conditions and an interval of 10 (continuous) time units.

We have run simulations for each triggering condition for 10 different initial conditions of ${ }^{1} x$ uniformly distributed on the circle centered at the origin of radius 10 . In all cases, we have selected $e(0,0)=0$ and $\eta(0,0)=10$, when relevant. The obtained average and minimum intertransmissions times over the ten simulations are summarized in Table I. We observe that, in all cases, the redesigned technique generates larger average inter-event times than the corresponding original one, thus fewer transmissions, while still guaranteeing desirable stability conditions. A similar improvement is seen for the minimum inter-event times, at the exception of the technique of Section VI.

The main purpose of this paper is to provide a unifying view of event-triggering design and proof techniques. Indeed, we concentrate on the "qualitative" properties of the eventtriggering mechanisms. This "qualitative" approach offers an opportunity to deal with "quantitative" issues, i.e., get tighter bounds on the gains, which have an impact on the number of transmissions. We acknowledge that the improvements reported in Table I on the average and minimum inter-event times are moderate. This can be explained by the fact that we do not strive to obtain the tightest bounds on the gains, We rather provide a principle here. Future work will be dedicated to tailor these bounds further to get even larger improvements.

\section{CONCLUSION}

A unifying emulation-based ETC design framework was proposed for a large class of nonlinear systems. The design consists of choosing the dynamics of auxiliary variables and jump and flow sets in such a manner that a small-gain condition holds. We showed that several well-known ETC schemes fit our framework and provided clear viewpoints on the essential differences and similarities of these ETC strategies. Moreover, we demonstrated the flexibility of the framework by providing redesigns of each of these schemes, which leads to the same stability guarantees as the original scheduler, however, typically using less transmissions. This was also illustrated through a numerical example. In future work, the framework can be generalized to encompass other (distributed) triggering conditions, as well as other control scenarios such as systems with disturbances and the output feedback control.

\section{REFERENCES}

[1] M. Abdelrahim, R. Postoyan, J. Daafouz, and D. Nešić. Stabilization of nonlinear systems using event-triggered output feedback controllers. IEEE Transactions on Automatic Control, 61:2682-2687, 2016.

\footnotetext{
${ }^{1}$ Although we know from [23] that a single initial condition is enough for the techniques of Section IV.
}

[2] A. Anta and P. Tabuada. To sample or not to sample: self-triggered control for nonlinear systems. IEEE Transactions on Automatic Control, 55(9):2030-2042, 2010.

[3] K.E. Årzén. A simple event-based PID controller. In Proceedings of the 14th IFAC World Congress, Beijing, China, volume 18, pages 423-428, 1999.

[4] K.J. Åström and B. Bernhardsson. Comparison of periodic and event based sampling for first-order stochastic systems. In Preprints 14th world congress of IFAC, volume J, pages 301-306, 1999.

[5] D.P. Borgers and W.P.M.H. Heemels. Event-separation properties of event-triggered control systems. IEEE Transactions on Automatic Control, 59:2644-2656, 2014.

[6] C. Cai and A. R. Teel. Characterizations of input-to-state stability for hybrid systems. Systems \& Control Letters, 58:47-53, 2009.

[7] T. Chen and B. A Francis. Optimal sampled-data control systems. Springer Science \& Business Media, 2012.

[8] V.S. Dolk, D.P. Borgers, and W.P.M.H. Heemels. Output-based and decentralized dynamic event-triggered control with guaranteed $L_{p^{-}}$ gain performance and Zeno-freeness. IEEE Transactions on Automatic Control, 62:34-49, 2017.

[9] V.S. Dolk, J. Ploeg, and W.P.M.H. Heemels. Event-triggered control for string-stable vehicle platooning. IEEE Transactions on Intelligent Transportation Systems, 18(12):3486-3500, 2017.

[10] A. Eqtami, D.V. Dimarogonas, and K.J. Kyriakopoulos. Eventtriggered control for discrete-time systems. In American Control Conference, Baltimore, U.S.A., pages 4719-4724, 2010.

[11] E. Garcia and P. J. Antsaklis. Model-based event-triggered control for systems with quantization and time-varying network delays. IEEE Transactions on Automatic Control, 58:422-434, 2013.

[12] A. Girard. Dynamic triggering mechanisms for event-triggered control. IEEE Transactions on Automatic Control, 60:1992-1997, 2015.

[13] R. Goebel, R.G. Sanfelice, and A.R. Teel. Hybrid dynamical systems: Modeling, Stability, and Robustness. Princeton University Press, 2012.

[14] W.P.M.H. Heemels, K.H. Johansson, and P. Tabuada. An introduction to event-triggered and self-triggered control. In IEEE Conference on Decision and Control, Maui, U.S.A., pages 3270-3285, 2012.

[15] J. Hespanha, D. Liberzon, and A.R. Teel. Lyapunov conditions for input-to-state stability of impulsive systems. Automatica, 44(11):27352744, 2008.

[16] B.A. Khashooei, D.J. Antunes, and W.P.M.H. Heemels. A consistent threshold-based policy for event-triggered control. IEEE Control Systems Letters, 2(3):447-452, 2018.

[17] D. Liberzon, D. Nešić, and A.R. Teel. Lyapunov-based smallgain theorems for hybrid systems. IEEE Transactions on Automatic Control, 59:1395-1410, 2014.

[18] S. Linsenmayer, D. V. Dimarogonas, and F. Allgower. Periodic event-triggered control for networked control systems based on nonmonotonic lyapunov functions. Automatica, 106:35-46, 2019.

[19] T. Liu and Z.-P. Jiang. Event-based control of nonlinear systems with partial state and output feedback. Automatica, 53:10-22, 2015.

[20] T. F. Liu and Z. P. Jiang. A small-gain approach to robust eventtriggered control of nonlinear systems. IEEE Transactions on Automatic Control, 60:2071-2085, 2015.

[21] J. Lunze and D. Lehmann. A state-feedback approach to event-based control. Automatica, 46:211-215, 2010.

[22] R. Postoyan, M.C. Bragagnolo, E. Galbrun, J. Daafouz, D. Nešić, and E.B. Castelan. Event-triggered tracking control of unicycle mobile robots. Automatica, 52:302-308, 2015.

[23] R. Postoyan, R.G. Sanfelice, and W.P.M.H. Heemels. Inter-event times analysis for planar linear event-triggered controlled systems. In IEEE Conference on Decision and Control, Nice: France, 2019.

[24] R. Postoyan, P. Tabuada, D. Nešić, and A. Anta. A framework for the event-triggered stabilization of nonlinear systems. IEEE Transactions on Automatic Control, 60:982-996, 2015.

[25] G.S. Seyboth, D.V. Dimarogonas, and K.H. Johansson. Event-based broadcasting for multi-agent average consensus. Automatica, 49:245$252,2013$.

[26] P. Tabuada. Event-triggered real-time scheduling of stabilizing control tasks. IEEE Transactions on Automatic Control, 52:1680-1685, 2007.

[27] W. Wang, R. Postoyan, D. Nešić, and W.P.M.H. Heemels. Periodic event-triggered control for nonlinear networked control systems. IEEE Transactions on Automatic Control, pages 620-635, 2020.

[28] X. Wang and M.D. Lemmon. Event design in event-triggered feedback control systems. In Proceedings of the 47th IEEE Conference on Decision and Control, pages 2105-2110, Cancun, Mexico, 2008. 